\title{
Recent developments in the treatment of small cell lung cancer
}

\author{
Birgitta I. Hiddinga ${ }^{1,6}$, Jo Raskin $\mathbb{1}^{2,6}$, Annelies Janssens ${ }^{2,3}$, Patrick Pauwels ${ }^{3,4,5}$ and \\ Jan P. Van Meerbeeck (i1) $2,3,5$
}

${ }^{1}$ Dept of Pulmonary Medicine and Tuberculosis, University Medical Centre Groningen, Groningen, The Netherlands. ${ }^{2}$ Dept of Thoracic Oncology, Antwerp University Hospital, Edegem, Belgium. ${ }^{3}$ University of Antwerp, Antwerp, Belgium. ${ }^{4}$ Dept of Pathology, Antwerp University Hospital, Edegem, Belgium. ${ }^{5}$ European Reference Network for rare and low prevalent lung diseases (ERN-LUNG), Frankfurt am Main, Germany. ${ }^{6}$ Both authors contributed equally.

Birgitta Hiddinga (b.i.hiddinga@umcg.nl)

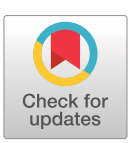

Copyright OThe authors 202

This version is distributed under the terms of the Creative Commons Attribution NonCommercial Licence 4.0. For commercial reproduction rights and permissions contact permissions@ersnet.org

Received: 29 Dec 2020

Accepted: 27 April 2021

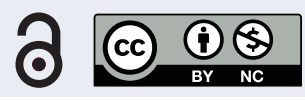

Shareable abstract (@ERSpublications)

Advances in radiotherapy, targeted treatment and immunotherapy are limited. Progress in treatment options are needed for the treatment of SCLC. Exome sequencing to identify targetable biomarkers could select patients who would benefit from certain therapies. https://bit.ly/3e7ATJ4

Cite this article as: Hiddinga BI, Raskin J, Janssens A, et al. Recent developments in the treatment of small cell lung cancer. Eur Respir Rev 2021; 30: 210079 [DOI: 10.1183/16000617.0079-2021].

\section{Abstract}

Small cell lung cancer (SCLC) comprises about 15\% of all lung cancers. It is an aggressive disease, with early metastasis and a poor prognosis. Until recently, SCLC treatment remained relatively unchanged, with chemotherapy remaining the cornerstone of treatment. In this overview we will highlight the recent advances in the field of staging, surgery, radiotherapy and systemic treatment. Nevertheless, the prognosis remains dismal and there is a pressing need for new treatment options. We describe the progress that has been made in systemic treatment by repurposing existing drugs and the addition of targeted treatment. In recent years, immunotherapy entered the clinic with high expectations of its role in the treatment of SCLC. Unravelling of the genomic sequence revealed new possible targets that may act as biomarkers in future treatment of patients with SCLC. Hopefully, in the near future, we will be able to identify patients who may benefit from targeted therapy or immunotherapy to improve prognoses.

\section{Introduction}

Small cell lung cancer (SCLC) is an extremely aggressive tumour type which accounts for about $15 \%$ of lung cancer cases [1, 2]. The cancer originates from neuroendocrine precursor cells and is characterised by its rapid growth and early metastasis, with more than $70 \%$ of patients presenting with metastasised disease [3]. Approximately $10-25 \%$ of patients have brain metastases at initial diagnosis, and an additional $40-50 \%$ will develop them during the course of their disease [3]. First-line treatment in metastatic SCLC consists of a combination of platinum and etoposide [4]. However, the majority of patients experience relapse within the first year of treatment, resulting in poor survival. Several agents or addition of a third drug have failed to show any improvement in outcomes. Even for patients without metastases at diagnosis, the curation rate remains low. Therefore, there is a high unmet need for therapies that could improve survival in patients with SCLC. The guidelines of the European Society of Medical Oncology (ESMO) and the American College of Chest Physicians (ACCP) endorsed by the American Society of Clinical Oncology (ASCO) have not been updated since 2013 [1, 5]. In this manuscript we will address the recent progress achieved in the field of staging, surgery, radiotherapy and systemic treatment, such as immunotherapy, since the landmark reviews published in the beginning of last decade $[6,7]$.

\section{Eighth tumour-node-metastasis classification}

The prognosis of SCLC depends on the tumour stage. Previously, the classifications limited disease (LD-SCLC) and extensive disease (ED-SCLC) were used, where limited disease was defined as disease confined to the ipsilateral hemithorax, which can safely be encompassed within a single radiation field [8]. The Union for International Cancer Control (UICC) tumour-node-metastasis (TNM) staging system was developed for nonsmall cell lung cancer (NSCLC), and edition 8 uses tumour size and the number of metastases and affected organs to estimate prognoses for the different stages of this disease [9]. In clinical 
practice, as well as in clinical trials, the distinction between LD-SCLC and ED-SCLC is still useful when deciding on a treatment plan.

Surgery in very limited SCLC

Surgery in SCLC is not widely accepted but can be considered for very small biopsy-proven tumours (very limited disease), cT1N0M0, with confirmed negative mediastinal staging. Most commonly, a surgically removed lung nodule of unknown origin turns out to be a small SCLC. In a systematic review, surgery was not supported in limited SCLC [10]. In several series data about correct staging and adjuvant therapy was unclear. Invasive mediastinal staging is mandatory. There is a tendency towards offering surgery for very small SCLC with negative lymph nodes, but concurrent chemoradiation is an alternative choice. Data about adjuvant radiotherapy and adjuvant chemotherapy are insufficient to offer strong recommendations [1]. Prospective studies are needed to define the role of surgery and adjuvant treatment of very small SCLC.

\section{Radiotherapy in LD-SCLC}

For LD-SCLC, which represents around 30\% of newly diagnosed SCLC, the standard treatment with curative intent consists of four cycles of platinum-doublet chemotherapy combined with radiotherapy, which improves overall survival compared with chemotherapy alone, even in elderly patients [11]. A concurrent approach is preferred, based on a median survival time of 27.2 months in the concurrent arm, compared with 19.7 months in the sequential arm of a Japanese trial [12]. Timing of radiotherapy is crucial, the shorter the overall treatment time, the better the 5-year overall survival (OS), with the start of radiotherapy preferably coinciding with the first or second cycle of chemotherapy [13, 14]. Despite the older phase 3 trials preferring twice-daily radiotherapy, once-daily radiotherapy is still the standard of care in most centres for practical reasons, and most patients do not qualify for twice-daily radiotherapy due to comorbidity and performance status [15].

The phase 3 trial CONVERT compared once-daily radiotherapy (66 Gy in 6.5 weeks) with twice-daily radiotherapy (45 Gy in 30 fractions in 3 weeks) concurrently with platinum/etoposide chemotherapy in localised SCLC [16]. The study was designed as a superiority trial, with the comparison of OS in both arms as the primary end-point, hoping to demonstrate the benefit of twice-daily irradiation. Of the 547 patients randomised, the two-year OS was 56\% in the twice-daily radiotherapy arm versus $51 \%$ in the conventional arm, which was not statistically significant. No difference in toxicity between both arms was reported.

Encouraged by the assumed noninferiority and safety of twice-daily radiotherapy in SCLC, a Scandinavian phase 2 trial compared the efficacy and tolerability of standard-dose $45 \mathrm{~Gy}$ in 30 fractions twice-daily and a high-dose 60 Gy in 40 fractions twice-daily, hoping to improve local control and thus survival [17]. Patients receiving the higher dose had a significantly longer 2 -year OS (73\% versus $46 \%$; $\mathrm{p}=0.002)$ and median OS (42 months versus 23 months; $\mathrm{p}=0.027$ ), without - unexpectedly - an increase in toxicity, and comparable tolerance for both arms [18]. The conclusion is that higher dose radiotherapy twice-daily in limited disease SCLC is feasible and tolerable compared with 45 Gy. The currently ongoing CALGB 30610 phase 3 trial is comparing once-daily high-dose thoracic radiotherapy (70 Gy/45 fractions) with standard twice-daily radiotherapy (45 Gy) [19]. The other experimental arm (61.2 Gy) was discontinued at interim analysis.

These trials may shed more light on the issue of the optimal radiation schedule for radiotherapy in locally advanced SCLC.

\section{Radiotherapy in ED-SCLC}

\section{Prophylactic cranial irradiation}

As the brain is a common site of distant failure in patients with SCLC, prophylactic cranial irradiation (PCI) is recommended in patients after curative treatment for limited stage disease [20]. In an older meta-analysis, the incidence of brain metastases decreased more than $25 \% 3$ years after PCI, with a doubling of survival, $42 \%$ versus $23 \%$ at 2 years [21]. However, these trials predate the current, more sensitive staging with MRI and positron emission tomography (PET) scans.

In a prospective trial, patients with stage IV SCLC with any response to chemotherapy, were randomised to PCI or no further treatment with the time to symptomatic brain metastases as the primary end-point [3]. Patients in the irradiated group had a lower risk of brain metastases at 1 year: the cumulative risk was $14.6 \%$ in the irradiated group and $40.4 \%$ in controls. The 1 -year survival rate was $27.1 \%$ in the irradiation group and $13.3 \%$ in controls. PCI appeared to be an effective add-on therapy, although the optimal total dose and fractionation schedule remains uncertain. Furthermore, the absence of systematic brain imaging before entering the study did raise concerns about the findings of this study. A Japanese phase 3 trial reassessed the efficacy of PCI in patients with metastasised SCLC with any response to chemotherapy [22]. Patients without 
brain metastases on MRI were randomised to PCI (25 Gy in 10 daily fractions of 2.5 Gy) or observation. After a planned interim analysis, the study was closed due to futility. The likelihood that PCI would be superior to observation at the end of the study was minimal. At 12 months follow-up, PCI reduced the incidence of brain metastases (32.9\% versus 59\%) but did not improve OS (48\% versus 54\% at 1 year).

The results of the Japanese PCI study challenge the benefits of PCI. Although PCI is generally well tolerated, patients experience fatigue, nausea, cognitive decline and ataxia [23]. These adverse events may be mild and transient, but could also be progressive and persistent with structural brain damage on MRI. Currently, the guidelines support PCI if patients respond to chemotherapy. The accepted radiation dose is $25 \mathrm{~Gy}$ in 10 fractions of $2.5 \mathrm{~Gy}$. There is no role for routine hippocampal sparing [24]. The results of the Japanese trial has led to the dismissal of PCI in many centres. The EORTC Lung Cancer Group is developing a randomised study of PCI versus watchful waiting with periodic brain MRI (PRIMALung). A randomised phase III study of the South-West Oncology Group is randomising patients to either MRI surveillance alone, or MRI surveillance with PCI [25].

\section{Consolidation thoracic radiotherapy}

Intrathoracic tumour control after chemotherapy remains a problem, as most patients have persistent disease, with disease progression within 1 year. Beneficial effects of thoracic radiotherapy were described in a retrospective series [26]. In a randomised phase 3, after completion of chemotherapy and PCI, thoracic irradiation (30 Gy in 10 fractions) was performed, resulting in an OS at 2 years of $13 \%$ versus $3 \%$ in controls [27]. The authors conclude that thoracic radiotherapy should be considered for patients with advanced disease with any response to chemotherapy. However, in clinical practice this advice is not implemented. Potentially, only patients with presenting symptoms of vena cava superior syndrome, central airway compression, or atelectasis of the lung may benefit from consolidation thoracic radiotherapy [27].

First-line systemic treatment in metastasised SCLC

SCLC is very sensitive to chemotherapy and treatment usually induces rapid responses. The current first-line treatment in ED-SCLC is platinum-based chemotherapy, four to six cycles of cis- or carboplatin plus etoposide in Europe and the United States, and platinum plus irinotecan in Japan [4]. Carboplatin is generally preferred over cisplatin due to its similar efficacy and lower toxicity [28]. However, the majority of patients experience a relapse within the first year of treatment: some of them during treatment (platinum-resistant), some within 90 days from the treatment interruption (platinum-refractory) and others 90 days or more after treatment stop (platinum-sensitive) [29]. In platinum-sensitive relapse, rechallenge with first-line chemotherapy is preferred $[1,5]$. Adding a third cytostatic agent to this therapeutic backbone has previously been shown not to result in a better outcome [6].

\section{Second-line therapy in SCLC}

Topotecan is the only drug that is formally approved as second-line treatment for SCLC and remains the standard of care. Oral topotecan had a response rate (RR) of $6-17 \%$ and a median survival of 25.9 weeks compared with a median survival of 13.9 weeks in a group that was assigned to best supportive care [30].

\section{Immunotherapy}

There is great interest in whether immune checkpoint inhibition (ICI) might play a role in the treatment of SCLC. The rationale for combining immunotherapy with chemotherapy in SCLC is the high mutational burden in this tumour, with potentially enhanced immunogenicity. Chemotherapy may stimulate the expression of tumoural antigens, priming the tumour for response to checkpoint inhibitory therapy.

Immunotherapy in adjuvant setting after curative chemoradiotherapy

Despite good initial responses to definitive treatment with curative chemoradiotherapy, outcomes remain poor, with a median progression free survival (PFS) of 15 months and OS of 25 months. The role of adjuvant immunotherapy in this setting was explored (table 1).

The STIMULI phase 2 study of maintenance nivolumab plus ipilimumab in LD-SCLC was conducted to evaluate whether adjuvant immunotherapy might improve outcomes after completion of concomitant chemoradiotherapy and PCI [31]. After randomisation of 153 patients of the 222 planned patients, the study was closed due to slow accrual. It did not meet its primary end-point of improving PFS (10.7 months versus 14.5 months). Treatment failure in the immunotherapy arm was mostly due to toxicity; treatment failure in the observation arm was due to disease progression.

In the ongoing phase 3 ADRIATIC trial, 600 patients with at least stable disease after concomitant chemoradiotherapy, with or without PCI, will be randomised 1:1:1 to receive durvalumab plus placebo, 
TABLE 1 Immunotherapy trials in small cell lung cancer

$\begin{array}{llcl}\text { Study [ref.] Trial design } & \text { Medication } & \begin{array}{c}\text { Number of } \\ \text { patients }\end{array} & \text { PFS (months) }\end{array}$

\begin{tabular}{|c|c|}
\hline \multicolumn{2}{|c|}{ Adjuvant in limited disease } \\
\hline STIMULI [31] & $\begin{array}{l}\text { Phase 2, } \\
\text { open-label }\end{array}$ \\
\hline ADRIATIC [32] & $\begin{array}{l}\text { Phase } 3, \mathrm{RCT} \text {, } \\
\text { double-blind }\end{array}$ \\
\hline \multicolumn{2}{|c|}{ First-line in metastasised SCLC } \\
\hline $\begin{array}{l}\text { NCT01331525 } \\
\text { [33] }\end{array}$ & $\begin{array}{l}\text { Phase 2, RCT, } \\
\text { double-blind }\end{array}$ \\
\hline $\begin{array}{l}\text { NCT00527735 } \\
\text { [34] }\end{array}$ & $\begin{array}{l}\text { Phase 2, RCT, } \\
\text { double-blind }\end{array}$ \\
\hline IDEATE [35] & $\begin{array}{l}\text { Phase } 3, \mathrm{RCT} \\
\text { double-blind }\end{array}$ \\
\hline $\begin{array}{c}\text { IMpower133 } \\
{[36,37]}\end{array}$ & $\begin{array}{l}\text { Phase } 3, \mathrm{RCT} \text {, } \\
\text { double-blind }\end{array}$ \\
\hline $\begin{array}{l}\text { CASPIAN } \\
{[39,40]}\end{array}$ & $\begin{array}{c}\text { Phase } 3, \mathrm{RCT} \\
\text { open-label }\end{array}$ \\
\hline
\end{tabular}

KEYNOTE-604 Phase 3, RCT,

[42] double-blind

ECOG-ACRIN Phase 2, RCT,

EA5161 [45] double-blind

REACTION [46] Phase 2, RCT,
1) Nivolumab+ipilimumab 2) Observation

1) Durvalumab+placebo

2) Durvalumab+tremelimumab 3) Placebo+placebo

patients
153 , closed
early
600 ,
recruiting

10.7

Was not met
1) Carboplatin/etoposide+ipilimumab

2) Carboplatin/etoposide+placebo
1) Carboplatin/paclitaxel+placebo (control arm)

2) Carboplatin/paclitaxel+ipilimumab followed by paclitaxel+carboplatin+placebo (concurrent arm)

3) Carboplatin/paclitaxel+placebo followed by carboplatin/paclitaxel+ipilimumab (phased arm)
1) Cisplatin/etoposide+ipilimumab
2) Cisplatin/etoposide+placebo

1) Carboplatin/etoposide+atezolizumab

2) Carboplatin/etoposide+placebo

1) Platinum/etoposide+durvalumab + tremelimumab

2) Platinum/etoposide+durvalumab

3) Platinum/etoposide

1) Platinum/etoposide+pembrolizumab

2) Platinum/etoposide+placebo

1) Platinum/etoposide+nivolumab

2) Platinum/etoposide

1) Platinum/etoposide+pembrolizumab

2) Platinum/etoposide+placebo
42

6.9

1 year PFS: $15.8 \%$

(6/35 patients)

130

5.2

3.9

5.2

9.9

1132
403
268

268

269

453

160

32

HR PFS

$4.6 \quad 11.0$

4.4

5.2

4.3

R PFS 0.78

HR PFS 0.84

5.1

HR PFS 0.78

$$
4.5
$$

4.3

HR PFS 0.75

5.5

4.6

HR PFS 0.68

5.4

4.7

HR PFS 0.84

1.4 irPFS 4.7

HR PFS 0.67

HR PFS 0.72

279

275

2) Nivolumab+ipilimumab 3) Placebo

1) Atezolizumab after response on chemotherapy and atezolizumab

2) Atezolizumab+(extra-)thoracic radiotherapy after response on chemotherapy and atezolizumab
138 phase 2 Primary end-point PFS in phase 2

186 phase 3 Primary end-point OS in phase 3

Progression after first-line chemotherapy
Checkmate
331 [50]
Phase 3, RCT
1) Nivolumab
2) Topotecan

Checkmate

032 [51]

Checkmate

KEYNOTE-028

[54]
Phase $1 / 2$, open-label

Phase $1 / 2$, open-label

Phase $1 b$, single
Nivolumab \pm ipilimumab in different dosages

1) Nivolumab $3 \mathrm{mg} \cdot \mathrm{kg}^{-1}$

2) Nivolumab $1 \mathrm{mg} \cdot \mathrm{kg}^{-1}+$ ipilimumab $3 \mathrm{mg} \cdot \mathrm{kg}^{-1}$

3) Nivolumab $3 \mathrm{mg} \cdot \mathrm{kg}^{-1}+$ ipilimumab $1 \mathrm{mg} \cdot \mathrm{kg}^{-1}$

Nivolumab monotherapy $3 \mathrm{mg} \cdot \mathrm{kg}^{-1}$ beyond third line

Pembrolizumab
569

216

7.5

8.4

1) ORR $10 \%$

2) ORR $23 \%$

3) ORR $19 \%$

ORR $11.9 \%$

9.7

ORR $33 \%$ 


\begin{tabular}{|c|c|c|c|c|c|}
\hline Study [ref.] & Trial design & Medication & $\begin{array}{l}\text { Number of } \\
\text { patients }\end{array}$ & PFS (months) & OS (months) \\
\hline $\begin{array}{l}\text { KEYNOTE-158 } \\
\text { [55] }\end{array}$ & $\begin{array}{l}\text { Phase 2, single } \\
\text { arm }\end{array}$ & Pembrolizumab & 107 & 2.0 & $\begin{array}{l}9.0 \text { (PDL-1 } 1^{+} 14.6 \text { months } \\
\text { and in PDL- } 1^{-} \\
7.7 \text { months) } \\
\text { ORR } 18.7 \% \text {, ORR in PDL- } 1^{+} \\
35.7 \% \text { and in PDL- } 1^{-} 6.0 \%\end{array}$ \\
\hline IFCT-1603 [56] & $\begin{array}{l}\text { Phase 2, } \\
\text { randomised, 2:1 }\end{array}$ & $\begin{array}{l}\text { 1) Atezolizumab } \\
\text { 2) Conventional chemotherapy }\end{array}$ & $\begin{array}{l}49 \\
24\end{array}$ & $\begin{array}{l}1.4 \\
4.3\end{array}$ & $\begin{array}{l}9.5 \\
8.7\end{array}$ \\
\hline BALTIC [57] & $\begin{array}{l}\text { Phase 2, } \\
\text { open-label }\end{array}$ & Durvalumab+tremelimumab & 21 & & ORR 9.5\% \\
\hline $\begin{array}{l}\text { MISP-MK3475 } \\
\text { [58] }\end{array}$ & $\begin{array}{l}\text { Phase 2, } \\
\text { single-arm, } \\
\text { open-label }\end{array}$ & Paclitaxel+pembrolizumab & 26 & 5.0 & $\begin{array}{c}9.1 \\
\text { ORR } 23.1 \%\end{array}$ \\
\hline
\end{tabular}

PFS: progression free survival; OS: overall survival; RCT: randomised controlled trial; SCLC: small cell lung cancer; HR: hazard ratio; irPFS: immune-related progression free survival; ORR: overall response rate; PDL-1: programmed death ligand-1.

durvalumab plus tremelimumab, or double placebo for a maximum of 24 months [32]. Primary end-points are PFS and OS for durvalumab, with or without tremelimumab, compared with placebo.

Currently, adjuvant ICI have no role in the treatment of locally advanced SCLC after completion of chemoradiotherapy with or without PCI.

\section{Immunotherapy in first-line metastasised SCLC}

Two pivotal phase 2 studies introduced immunotherapy to SCLC, combining the CTLA-4 inhibitor ipilimumab with first-line chemotherapy [33, 34] (table 1). In the first study, the primary end-point of 1-year PFS was not met [33]. The second phase 2 study showed a slightly better outcome for patients treated in the phased ipilimumab versus concurrent ipilimumab with chemotherapy [34].

In the large phase 3 study, IDEATE, patients were randomly assigned to receive chemotherapy (platinum/ etoposide) plus ipilimumab or placebo every 3 weeks for a total of four doses in a phased schedule [35]. The primary end-point OS was not met, with higher toxicity in the chemotherapy/ipilimumab arm. Ipilimumab may not be effective without corresponding T-cell activation in the tumour environment.

The IMPOWER-133 study was designed to evaluate the safety and efficacy of atezolizumab versus placebo in combination with carboplatin/etoposide in 403 treatment-naive participants with metastasised SCLC [36]. The hazard ratio (HR) for disease progression or death was $0.77(p=0.02)$. The addition of atezolizumab to chemotherapy in the first-line treatment of metastasised SCLC resulted in a longer OS (33.5\% long-term survivors versus $20.4 \%$ for placebo) and PFS than chemotherapy alone [37]. Although only $43 \%$ of tumour specimens were evaluable for programmed death ligand-1 (PD-L1), neither PD-L1 nor the tumour mutational burden (TMB) were found to discriminate long-term survivors. Chemotherapy plus atezolizumab had a comparable safety profile to chemotherapy alone, and did not result in impaired quality of life [38]. Atezolizumab has been approved for registration by both the US Federal Drugs Administration (FDA) and the European Medicines Agency (EMA) [2].

In the phase 3 CASPIAN-trial, the addition of durvalumab and tremelimumab to chemotherapy was also evaluated in treatment-naive patients with metastasised SCLC [39]. Patients were randomly assigned (in a 1:1:1 ratio) to durvalumab plus chemotherapy, durvalumab/tremelimumab plus chemotherapy, or platinum/ etoposide alone. First-line durvalumab plus chemotherapy significantly improved OS (22\% after 24 months) in patients with advanced SCLC compared with chemotherapy alone. No additional benefit of tremelimumab was observed [40]. However, three times more patients derived long-term benefit when treated with durvalumab plus chemotherapy compared with chemotherapy alone [41]. Patients in all arms with a PFS $>12$ months had improved overall response rate (ORR), duration of response and OS compared with the PFS $<12$ months subgroup. Assessment of the characteristics that lead to long-term benefit is ongoing.

In the phase 3 Keynote-604 study, the primary end-point, OS, was prolonged in the pembrolizumab/ chemotherapy arm compared with chemotherapy alone [42]. Although PFS improved in the 
pembrolizumab arm, the significance threshold was not met (HR 0.8, 95\% CI 0.61-0.98). ORR was 71\% in the pembrolizumab arm and $62 \%$ for placebo. Adding pembrolizumab to chemotherapy did not decrease quality of life $[43,44]$.

These phase 3 studies showed an improved OS and PFS by adding immunotherapy to first-line chemotherapy, with an acceptable safety profile and quality of life, which supports this regimen as standard of care.

The phase 2 ECOG-ACRIN EA5161-study randomised between platinum/etoposide plus maintenance nivolumab and platinum/etoposide plus observation [45]. The median PFS (primary end-point) and OS were clinically significant, 5.5 months and 11.3 months in the nivolumab plus chemotherapy arm versus 4.6 months and 9.3 months in the chemotherapy arm, respectively.

In the phase 2 REACTION-study, chemotherapy with or without pembrolizumab in first-line treatment showed similar results with a not significant PFS of 5.4 months versus 4.7 months [46].

\section{Maintenance immunotherapy after first-line chemotherapy}

The results of maintenance immunotherapy after completion of first-line chemotherapy are disappointing $[47,48]$. In the phase 3 CheckMate-451 trial, patients with responses after completion of first-line chemotherapy were randomised between nivolumab, nivolumab with ipilimumab, or placebo as maintenance therapy [48]. Maintenance immunotherapy did not improve OS, but favourable PFS suggests that some patients could have benefited from maintenance therapy. In a phase 2 study with pembrolizumab, PD-L1 could be assessed in 30 of 45 patients and was positive (PD-L1 expression $>1 \%$ ) in three patients [47], having a PFS of 10, 11 and 13 months. Each unit increase in baseline circulating tumour cells correlated with worse PFS ( $\mathrm{p}=0.052$; adjusted for brain metastases, age and sex). Biomarkers to identify the patients most likely to benefit from immunotherapy in the maintenance setting are also lacking.

The RAPTOR trial is evaluating a new strategy of whether thoracic radiotherapy plus maintenance atezolizumab after response on first-line atezolizumab and chemotherapy is better than atezolizumab maintenance alone in patients with metastasised SCLC [49].

\section{Immunotherapy in second-line therapy and beyond}

Nivolumab has been investigated in the phase 3 CheckMate-331 trial versus topotecan or amrubicin as second-line therapy after progression on standard chemotherapy [50]. The study resulted in a median OS of 7.5 months with nivolumab versus 8.4 months with chemotherapy (HR 0.86, 95\% CI 0.72-1.04). Immunotherapy in second-line therapy showed no improvement in therapy for SCLC.

Ongoing approaches include combinations of anti-CTLA-4 (ipilimumab and tremelimumab) and anti-PD-(L)1 therapy (nivolumab, pembrolizumab, atezolizumab and durvalumab). In the CheckMate-032 trial, nivolumab/ ipilimumab, and nivolumab alone, were evaluated in pretreated patients with SCLC [51, 52]. The responses were fast and durable for patients with relapsed SCLC, regardless of platinum sensitivity or PD-L1 status. Nivolumab monotherapy is approved in the United States as third-line or later based on the pooled data of this trial. In a separate analysis of the pooled nivolumab monotherapy cohort in CheckMate-032, the ORR was $21.3 \%$ in patients with a high TMB versus $4.8 \%$ in those with a low TMB [53]. In the nivolumab plus ipilimumab arm the efficacy was also enhanced in the high TMB group, suggesting TMB may nevertheless have a role as a biomarker for immunotherapy in SCLC, but this has to be further explored.

Several phase 1 and 2 studies evaluated diverse ICIs, which all failed to show efficacy in patients with relapsed SCLC [54-58]. Although in a selected patient category with PD-L1 expression, a slightly better ORR was noted.

\section{Biomarkers in immunotherapy}

PD-L1 and TMB are also emerging as biomarkers of response to immune checkpoint inhibitors in various cancer types, including SCLC [53, 59]. Most SCLC tumours seem to lack PD-L1 expression [60]. A recently conducted study speculates that only $2 \%$ of patients with SCLC exhibit amplification of the gene CD274, which encodes for PD-L1 expression, and only this small subgroup may be susceptible to ICI [61]. Currently, PD-L1 has no clinical application in SCLC. Some evidence exists that high TMB may be associated with a response to ICI, but large phase 3 studies in patients with first-line metastasised SCLC failed to confirm this [36, 60]. The predictive role of TMB in SCLC has to be defined. The diagnosis of SCLC is made on small biopsies and the evaluation of PD-L1 in tumour tissue is challenging. 
Further investigation is ongoing to assess biomarkers such as TMB in tissue and in blood [62]. Liquid biopsies are less invasive for patients [63].

\section{Recommendations in clinical practice}

Patients with SCLC still have a poor prognosis and little progress has been made during the last few decades. Surgery for very small SCLC after adequate mediastinal staging seems feasible, but the role of adjuvant chemotherapy is still undecided. Radiotherapy is of additional value in all stages of the disease. For treatment with a curative intent, the proposed radiotherapy schedule of twice-daily irradiation, 45 Gy in 30 fractions seems feasible, and also for elderly patients. From a pragmatic perspective, once-daily radiotherapy should be considered when twice-daily radiotherapy is impractical. After completion of chemoradiotherapy, current guidelines support PCI in case of any response to chemotherapy, with most commonly a radiation dose of 25 Gy in 10 fractions of 2.5 Gy. Discussion about the benefits of PCI, both in the localised and metastasised setting is ongoing. Another option is watchful waiting with periodic brain MRI. Thoracic radiotherapy should be considered for patients with advanced disease who have any response to chemotherapy and present with symptoms such as vena cava superior syndrome, central airway compression and atelectasis of the lung.

Chemotherapy remains the mainstay of the treatment of advanced SCLC. The addition of checkpoint inhibition to the standard backbone chemotherapy has added a modest but significant improvement in outcomes, but predictive biomarkers are yet to come.

\section{Future perspectives}

Unravelling the genomics of SCLC and the subsequent discovery of biomarkers is crucial for treatment selection [64]. Whole exome sequencing may help in identifying these biomarkers and targets [65, 66]. For example, in SCLC, loss of TP53 and RB1 occurs most frequently, which results in proliferation and replication stress in SCLC and is associated with early metastasis and rapid resistance against chemotherapy [64]. Amplification of the MYC family of oncogenes occurs in 20\% of SCLC and is associated with shorter survival [67]. MYC downregulation suppresses tumour growth.

The clinical relevance of biomarking SCLC lies in preferential targeting of different routes or combinations thereof, or even combining treatment modalities.

\section{Targeting the DNA damage repair pathway}

Recent preclinical studies identified predictive biomarkers of response to DNA damage repair (DDR)-targeted therapies in SCLC. Repair proteins such as poly-(ADP)-ribose polymerase (PARP), WEE1, ataxia-telangiectasia-mutated-and-Rad3-related kinase (ATR) and its major downstream effector checkpoint kinase 1 (CHK1) seems attractive to target [68]. PARP, WEE1, ATR and CHK1 prevent entry of cells with damaged or incomplete replicated DNA into mitosis and thus suppress replication stress. Inhibition of these proteins results in cell death. AZD1775 (adavosertib) is a highly selective, potent small molecule inhibitor of WEE1. This small molecule is being tested in several phase 1 and 2 studies, both alone and in combination with PARP inhibition or chemotherapy [69, 70].

\section{Veliparib}

Veliparib is a PARP-inhibitor; a small molecule that traps the DNA-repair enzyme PARP on DNA single-strand breaks and blocks its catalytic activity, thus potentially enhancing the damage to DNA caused by chemotherapy. The randomised phase 2 trial of platinum/etoposide plus veliparib or placebo showed a median PFS of 6.1 months versus 5.5 months in favour of veliparib [71]. Recent genomic sequencing led to the identification of Schlafen 11 (SLFN11), a predictive biomarker for sensitivity to PARP inhibition in monotherapy in SCLC [72]. SLFN11 expression is high in SCLC and decreases significantly after treatment with veliparib. Prospective validation of the potential of SLFN11 as a predictive biomarker in patients treated with veliparib is warranted.

\section{Temozolomide}

Temozolomide (Temodal ${ }^{\mathrm{TM}}$ ) is a triazene derivative causing DNA breakage by adding an alkyl group to the guanine base of DNA. It is an oral drug, well tolerated and not very toxic, with excellent penetration into the central nervous system [73]. Beneficial effects of treatment with temozolomide in patients with SCLC were reported, especially in a subgroup associated with the presence of MGMT promoter methylation, although the difference did not meet statistical significance [74]. The RR in an unselected group was $22 \%$ in second-line, $19 \%$ in third-line, and $38 \%$ in patients with brain metastases. Temozolomide is strongly synergistic with PARP inhibition by preventing the repair of alkylated bases [75]. A phase 2 trial evaluated temozolomide with either veliparib or placebo in patients with relapsed SCLC [76]. Translational objectives 
included PARP-1 and SLFN11 immunohistochemical expression, MGMT promoter methylation and circulating tumour cell quantification. Four-month PFS and median OS did not differ between the two arms, but a significant improvement in ORR, which was a secondary end-point, was observed with temozolomide/ veliparib compared with temozolomide/placebo (39\% versus 14\%). In patients with SLFN11-positive tumours treated with temozolomide/veliparib a significantly prolonged PFS (5.7 versus 3.6 months) and OS (12.2 versus 7.5 months) were observed, suggesting PARP-inhibitor sensitivity as a promising biomarker in SCLC. These findings were confirmed in a single-arm trial with olaparib, reporting a lesser effect in platinum-resistant disease [77].

Lurbinectedin

Lurbinectedin is a selective inhibitor of oncogenic transcription, promoting tumour cell death and normalising the tumour microenvironment [78]. In the phase 2 basket trial, 105 patients were treated with lurbinectedin after failure of platinum-based chemotherapy [79]. Activity of lurbinectedin appeared to be greater in patients with a longer chemotherapy-free interval: ORR of $45 \%$ versus $22 \%$ in the platinum-resistant arm. Among all patients, median PFS was 3.5 months (2.6 versus 4.6 months) and the median OS was 9.3 months (5.0 versus 11.9 months). The most common toxicities were leukopenia and neutropenia. In the phase 3 ATLANTIS-trial comparing lurbinectedin/doxorubicin with either topotecan or cyclophosphamide/doxorubicin/vincristine in second-line metastasised disease, the primary end-point OS was not met [80].

Drug resistance is often a problem of the DDR network. AXL is recognised as the key determinant in both intrinsic and acquired resistance to chemotherapeutic, immunotherapeutic and molecularly targeted agents in SCLC by epithelial-to-mesenchymal transformation (EMT) [81]. High levels of AXL and EMT predict resistance to PARP, ATR and WEE1 targeting. AXL-inhibition induces DNA damage and replication stress and promotes sensitivity to PARP and ATR inhibitors [82, 83]. In addition SLFN11 holds promise as a potential biomarker, while cells with low levels of SLFN11 were more sensitive to AXL/ATR inhibition [84].

Strategies on combinations of DDR-inhibitors or targeting multiple pathways are to be explored. Inhibitors of the DDR pathway confers a synergistic effect on immunotherapy, radiotherapy and chemotherapy; for example, temozolomide [83, 85]. PARP inhibition enhances the effect of radiotherapy in SCLC in a preclinical model [86]. A continuing challenge in SCLC is the intra-tumour heterogeneity. Blocking various routes of growth to tackle the tumour might be the answer.

Targeting the genomic and epigenomic alterations

Potential targetable genomic alterations are mutations in PTEN or RET, and amplifications of fibroblast growth factor receptor 1 (FGFR1) [87-89]. The latter are present in 6\% of SCLC [89]. RET mutations are found in 1-2\% of SCLC [88]. RET mutated SCLC also seems to express MYC more often. Inactivation of RB1 leads to overexpression of enhancer of zeste homolog 2 (EZH2) which promotes tumour genesis in SCLC [90]. In this process, downregulation of SLFN11 due to overexpression of EZH2 makes SCLC resistant to chemotherapy [91]. The combination of an EZH2 inhibitor and chemotherapy, such as cisplatin or temozolomide, can circumvent resistance by preventing loss of SLFN11. Expression of EZH2 can act as biomarker and therapeutic target in SCLC.

Pazopanib

Pazopanib is a tyrosine kinase inhibitor that inhibits downstream signalling of vascular endothelial growth factor receptor (VEGFR)-1, -2 and -3. These targets are considered interesting given the importance of neoangiogenesis in SCLC and the role of VEGF overexpression in development of resistance to chemotherapy. In a multicentre, single-arm phase 2 trial, 58 patients were treated with pazopanib in second-line [92]. The platinum-refractory cohort was closed early due to futility. Median PFS and OS in cohort the platinum-sensitive cohort were 3.7 months and 8.0 months, respectively, with an ORR of 13.8\%. Pazopanib is a tolerable and effective salvage treatment in patients with platinum-sensitive SCLC. However, with its modest effect, pazopanib will likely play no role except in FGFR1-amplified SCLC [93].

Alisertib

Alisertib is a selective oral Aurora kinase A (AURK) inhibitor. AURKs are mitotic regulators required for normal cell proliferation [94]. Errors in mitosis may either lead to cell death, or to aneuploidy and a mitotic dysregulator might offer a therapeutic target with relative sparing of normal cells. A phase 1-2 study in five cancer types reported a promising ORR of $21 \%$ for the SCLC arm [95]. This was further explored in a phase 2 trial randomising between paclitaxel plus alisertib (based on preclinical evidence of synergy) or paclitaxel plus placebo, and reported a median PFS of 3.3 months for the alisertib arm, which 
was not significantly better than placebo (the ORR of 22\% was confirmed) [96]. In a subset of patients with high MYC expression, PFS for the alisertib arm was significantly better (4.6 months versus 2.3 months), but as this analysis was not part of the protocol, this will not be further explored. In spite of this discovery of MYC as a potential biomarker in SCLC, development of alisertib was halted.

\section{Targeting the immune system and genomic instability}

Defects in the DDR pathway have been associated with enhanced responses to immune checkpoint blockade due to high TMB and genomic instability [97]. Recently, it was found that co-targeting DDR proteins such as PARP and CHK1 can increase expression of PD-L1 and antitumour immune response of anti-PD-L1 in SCLC [98, 99]. These findings suggest that DDR targeting in combination with immunotherapy could be successful.

\section{Drug-delivery challenges}

Novel drug-delivery systems such as antibody drug conjugates (ADC) bring medication in the vicinity of the tumour and should help to target tumour cells without damaging healthy cells.

\section{Rovalpituzumab-tesirine}

An initially very promising target was delta-like protein 3 (DLL3), a Notch ligand that is highly expressed in about two-thirds of SCLC [100]. Activation of Notch inhibits the growth of SCLC-cells in vitro. Rovalpituzumab-tesirine (Rova-T) is an ADC that binds to DLL3, with an ORR of $18 \%$ in the phase 1 trial, which increased to 39\% in high ( $\geqslant 50 \%$ ) DLL3-expressors [101]. The subsequent phase 2 TRINITY trial selected DLL3-expressing tumours in extensively pre-treated patients (at least two lines) but found only an ORR of $12.4 \%$ and an OS of 5.7 months, with DLL3-high patients only performing slightly better than DLL3-non high patients [102]. Grade 3-5 toxicity was found in $63 \%$, with $10 \%$ grade 5 toxicity. We may conclude that Rova-T is the first targeted agent in SCLC to target DLL3, but results are disappointing. As a result of this, the product was withdrawn and ongoing studies TAHOE (versus topotecan) and MERU (maintenance after chemotherapy) closed prematurely [103, 104].

Recently, a definition of four molecular subsets of SCLC have been proposed: acheate-scute homolog 1 (ASCL1), neurogenic differentiation factor 1 (NEUROD1), yes-associated protein 1 (YAP1) and POU domain class 2 homebox 3 (POU2F3) [105, 106]. These molecular subtypes appear to be associated with distinct expression profiles and possible therapeutic sensitivities [107]. For instance, both ASCL1-high and NEUROD1-high neuroendocrine subtypes are characterised by marked expression of insulinoma-associated protein 1 (INSM1), a marker of super-enhanced landscapes in SCLC [105]. SCLC with high NEUROD1 expression have high MYC expression [108]. High MYC expression and amplification predicts sensitivity to AURK and CHK1 inhibitors. In combination with chemotherapy, it strongly suppresses tumour progression and increases survival [67]. MYC activates Notch, so ASCL1 and NEUROD1 subtypes could benefit from DLL3 inhibitors such as Rova-T. The combination of Rova-T and immunotherapy, however, was not well tolerated despite antitumour activity in third-line and beyond [109]. Subtypes with low ASCL1, NEUROD1 and POU2F3 expression act as inflamed SCLC and can benefit from the addition of immunotherapy to chemotherapy [110].

\section{Concluding remarks}

The histopathology and tumour biology of SCLC is complex. Simple strategies to target the tumour are not successful in achieving a long survival advantage. Several approaches have the potential to overcome the well-known treatment failures of the last decades.

Firstly, recent studies have shown progress in finding biomarkers to serve as targets for treatment. Extensive exome sequencing in patients with SCLC is the future to create a landscape of predictive biomarkers in SCLC. Epigenetic alterations, gene amplifications and mutations can act as biomarkers in this context. Consequently, biomarker-driven patient selection is needed to stratify patients for treatments. Distinct molecular subtypes appear to be associated with therapeutic sensitivities. The key to success lies in the treatment combination or targeting dual pathways that have additional or synergistic effects. Overcoming intra-tumour heterogeneity is an extra hurdle where combination therapy, concomitantly or sequentially, is probably a "conditio sine qua non". Secondly, it is expected that adding other treatment modalities, such as radiotherapy or immunotherapy, to biomarker-driven drug combinations will have synergistic effects to overcome resistance mechanisms. Lastly, novel drug-delivery systems should help to target tumour cells while preventing deleterious effects due to interaction with healthy cells. 
With possible biomarkers having been discovered, the design of future trials should allow the study of a targeted treatment in a biomarker enriched population. Importantly, referrals of patients for clinical trials with biomarker-selected targeted treatments is warranted to improve the prognosis for patients with SCLC.

Provenance: Submitted article, peer reviewed

Conflict of interest: B.I. Hiddinga has nothing to disclose. J. Raskin reports personal fees from Lilly, Pfizer and BMS, and non-financial support from Roche, outside the submitted work. A. Janssens has nothing to disclose. P. Pauwels has nothing to disclose. J.P. van Meerbeeck has nothing to disclose.

\section{References}

1 Früh, M, De Ruysscher D, Popat S, et al. Small-cell lung cancer (SCLC): ESMO Clinical Practice Guidelines for diagnosis, treatment and follow-up. Ann Oncol 2013; 24: Suppl. 6, vi99-vi105.

2 Kalemkerian GP, Loo BW, Akerley W, et al. NCCN Guidelines insights: small cell lung cancer - clinical practice guidelines in oncology, version 2.2018. J Natl Compr Canc Netw 2018 16: 1171-1182.

3 Slotman B, Faivre-Finn C, Kramer G, et al. Prophylactic cranial irradiation in extensive small-cell lung cancer. N Engl J Med 2007; 357: 664-672.

4 Mascaux C, Paesmans M, Berghmans T, et al. A systematic review of the role of etoposide and cisplatin in the chemotherapy of small cell lung cancer with methodology assessment and meta-analysis. Lung Cancer 2000; 30: 23-36.

5 Rudin CM, Ismaila N, Hann CL, et al. Treatment of small-cell lung cancer: American Society of Clinical Oncology Endorsement of the American College of Chest Physicians Guideline. J Clin Oncol 2015; 33: 4106-4111.

$6 \quad$ Van Meerbeeck JP, Fennell DA, De Ruysscher DKM. Small-cell lung cancer. Lancet 2011; 378: 1741-1755.

7 Demedts IK, Vermaelen KY, Van Meerbeeck JP. Treatment of extensive-stage small cell lung carcinoma: current status and future prospects. Eur Respir J 2010; 35: 202-215.

8 Stahel R, Ginsberg R, Havemann K, et al. Staging and prognostic factors in small cell lung cancer: a consensus report. Lung Cancer 1989; 5: 119-126.

9 Nicholson AG, Chansky K, Crowley J, et al. The International Association for the Study of Lung Cancer Staging Project: proposals for the revision of the clinical and pathologic staging of small cell lung cancer in the forthcoming eight edition of the TNM classification for lung cancer. J Thorac Oncol 2015; 11: 300-311.

10 Barnes H, See K, Barnett S, et al. Surgery for limited-stage small-cell lung cancer. Cochrane Database Syst Rev 2017; 4: CD011917.

11 Corso CD, Rutter CE, Park HS, et al. Role of chemoradiotherapy in elderly patients with limited-stage small-cell lung cancer. J Clin Oncol 2015; 33: 4240-4246.

12 Takada M, Fukuoka M, Kawahara M, et al. Phase III study of concurrent versus sequential thoracic radiotherapy in combination with cisplatin and etoposide for limited-stage small-cell lung cancer: results of the Japan Clinical Oncology Group Study 9104. J Clin Oncol 2002; 20: 3054-3060.

13 De Ruysscher D, Pijls-Johannesma M, Bentzen SM, et al. Time between the first day of chemotherapy and the last day of the chest radiation is the most important predictor of survival in limited-disease small-cell lung cancer. J Clin Oncol 2006; 24: 1057-1063.

14 De Ruysscher D, Lueza B, Le Péchoux C, et al. Impact of thoracic radiotherapy timing in limited-stage small-cell lung cancer: usefulness of the individual patient data meta-analysis. Ann Oncol 2016; 27: 1818-1828.

15 Turrisi AT, Kim K, Blum R, et al. Twice-daily compared with once-daily thoracic radiotherapy in limited small-cell lung cancer treated concurrently with cisplatin and etoposide. N Engl J Med 1999; 340: 265-271.

16 Faivre-Finn C, Snee M, Ashcroft L, et al. Concurrent once-daily versus twice-daily chemoradiotherapy in patients with limited-stage small-cell lung cancer (CONVERT): an open-label, phase 3, randomized, superiority trial. Lancet Oncol 2017; 18: 1116-1125.

17 Grønberg, BH, Killingberg KT, Øystein F, et al. Randomized phase II trial comparing the efficacy of standard-dose with high-dose twice-daily thoracic radiotherapy (TRT) in limited disease small-cell lung cancer (LD-SCLC). J Clin Oncol 2020; 38: 15_suppl.9007.

18 Grønberg BH, Killingberg KT, Stokke K, et al. Patient reported outcomes from a randomized phase II trial comparing standard-dose with high-dose twice-daily thoracic radiotherapy in limited stage small cell lung cancer. Ann Oncol 2020; 31: 17830.

19 ClinicalTrials.gov. Radiation therapy regimens in treating patients with limited-stage small cell lung cancer receiving cisplatin and etoposide. https://clinicaltrials.gov/ct2/show/NCT00632853. Date last accessed: March 4, 2021. Date last updated: January 11, 2021. 
Auperin A, Arriagada R, Pignon JP, et al. Prophylactic cranial irradiation for patients with small-cell lung cancer in complete remission. Prophylactic Cranial Irradiation Overview Collaborative Group. N Engl J Med 1999; 341: 476-484.

Patel S, Macdonald OK, Suntharalingam M, et al. Evaluation of the use of prophylactic cranial irradiation in small cell lung cancer. Cancer 2009; 115: 842-850.

Takahashi T, Yamanaka T, Seto T, et al. Prophylactic cranial irradiation versus observation in patients with extensive-disease small-cell lung cancer: a multicentre, randomised, open-label, phase 3 trial. Lancet Oncol 2017; 18: 663-671.

Slotman B, Mauer ME, Bottomley A, et al. Prophylactic cranial irradiation in extensive disease small-cell lung cancer: short-term health-related quality of life and patient reported symptoms - results of an international phase III randomized controlled trial by the EORTC Radiation Oncology and Lung Cancer Groups. J Clin Oncol 2008; 27: 78-84.

Belderbos JSA, De Ruysscher DKM, De Jaeger K, et al. Phase III randomized trial of prophylactic cranial irradiation with or without hippocampus avoiding in SCLC (NCT01780675). J Thorac Oncol 2020; 16: 840-849. ClinicalTrials.gov. Testing whether the use of brain scans alone instead of brain scans plus preventive brain radiation affects lifespan in patients with small cell lung cancer. https://clinicaltrials.gov/ct2/show/ NCT04155034. Date last accessed: September 24, 2020. Date last updated: April 30, 2021.

Zhu $\mathrm{H}$, Zhou Z, Wang $\mathrm{Y}$, et al. Thoracic radiotherapy improves the overall survival of patients with extensive-stage small cell lung cancer with distant metastasis. Cancer 2011; 117: 5423-5431.

Slotman B, Van Tinteren H, Praag JO, et al. Use of thoracic radiotherapy for extensive stage small-cell lung cancer: a phase 3 randomised controlled trial. Lancet 2015; 385: 36-42.

Rossi A, Di Maio M, Chiodini P, et al. Carboplatin- or cisplatin-based chemotherapy in first-line treatment of small-cell lung cancer: the COCIS meta-analysis of individual patient data. J Clin Oncol 2012; 30: 1962.

Ardizzoni A, Tiseo M, Boni L. Validation of standard definition of sensitive versus refractory relapsed small cell lung cancer: a pooled analysis of topotecan second-line trials. Eur J Cancer 2014; 50: 2211-2218.

O'Brien MER, Ciuleanu TE, Tsekov $\mathrm{H}$, et al. Phase III trial comparing supportive care alone with supportive care with oral topotecan in patients with relapsed small-cell lung cancer. J Clin Oncol 2006; 24: 5441-5447.

Peters S, Pujol JL, Dafni U, et al. Consolidation ipilimumab and nivolumab vs observation in limited stage SCLC after chemo-radiotherapy - results from the ETOP/IFCT 4-12 STIMULI trial. Ann Oncol 2020; 31: LBA84.

Senan S, Okamoto I, Lee GW, et al. Design and rationale for a phase III, randomized, placebo-controlled trial of durvalumab with or without tremelimumab after concurrent chemoradiotherapy for patients with limited-stage small-cell lung cancer: the ADRIATIC Study. Clin Lung Cancer 2020; 21: e84-e88.

Arriola E, Wheater M, Galea I, et al. Outcome and biomarker analysis from a multicentre phase 2 study of ipilimumab in combination with carboplatin and etoposide as first-line therapy for extensive-stage SCLC. J Thorac Oncol 2016; 11: 1511-1521.

Reck M, Bondarenko I, Luft A, et al. Ipilimumab in combination with paclitaxel and carboplatin as first-line therapy in extensive-disease-small-cell lung cancer: results from a randomized, double-blind, multicentre phase 2 trial. Ann Oncol 2013; 24: 75-83.

Reck M, Luft A, Szczesna A, et al. Phase III randomized trial of ipilimumab plus etoposide and platinum versus placebo plus etoposide and platinum in extensive-stage small-cell lung cancer. J Clin Oncol 2016; 34: 3740-3748. Horn L, Mansfield AS, Szczesna A, et al. First-line atezolizumab plus chemotherapy in extensive-stage small-cell lung cancer. N Engl J Med 2018; 379: 2220-2229.

Liu SV, Horn L, Mok TS, et al. IMpower 133: characterisation of long-term survivors treated first-line with chemotherapy - atezolizumab in extensive-stage small cell lung cancer. Ann Oncol 2020; 31: 1718MO.

Mansfield AS, Kazarnowicz A, Karaseva N, et al. Safety and patient-reported outcomes of atezolizumab, carboplatin, and etoposide in extensive-stage small-cell lung cancer (IMpower133): a randomized phase I/III trial. Ann Oncol 2020; 31: 310-317.

39 Paz-Ares L, Dvorkin M, Chen Y, et al. Durvalumab plus platinum-etoposide versus platinum-etoposide in first-line treatment of extensive-stage small-cell lung cancer (CASPIAN): a randomised, controlled, open-label, phase 3 trial. Lancet 2019; 394: 1929-1939.

Paz-Ares LG, Dvorkin M, Chen Y, et al. Durvalumabıtremelimumab+platinum-etoposide in first-line extensive-stage SCLC (ES-SCLC): updated results from the phase III CASPIAN study. J Clin Oncol 2020; $38: 15$ suppl.9002.

Goldman JW, Garassino MC, Chen Y, et al. Durvalumab - tremelimumab+platinum - etoposide in first-line extensive-stage small cell lung cancer: characterization of long-term clinical benefit of tumour mutational burden in CASPIAN. Ann Oncol 2020; 31: LBA86.

Rudin CM, Awad MM, Navarro A, et al. KEYNOTE-604: pembrolizumab (pembro) or placebo plus etopide and platinum (EP) as first-line therapy for extensive-stage (ES) small-cell lung cancer (SCLC). J Clin Oncol 2020; 38: 15 suppl.9001.

Kim HR, Awad MM, Navarro A, et al. Health-related quality of life in KEYNOTE604: pembrolizumab (pembro) or placebo added to etoposide and platinum as first-line therapy for extensive-stage small cell lung cancer. Ann Oncol 2020; 31: 1782MO. 
Rudin CM, Awad MM, Navarro A, et al. Pembrolizumab or placebo plus etoposide and platinum as first-line therapy for extensive-stage small-cell lung cancer: randomized, double-blind, phase III KEYNOTE-604 study. J Clin Oncol 2020; 38: 2369-2379.

Leal T, Wang Y, Dowlati A, et al. Randomized phase II clinical trial of cisplatin/carboplatin and etoposide (CE) alone or in combination with nivolumab as frontline therapy for extensive-stage small cell lung cancer (ES-SCLC): ECOG-ACRIN EA5161. J Clin Oncol 2020; 38: 15 suppl.9000. Besse B, Menis J, Bironzo P, et al. REACTION: a phase II study of etoposide and cis/carboplatin with or without pembrolizumab in untreated extensive small cell lung cancer. Ann Oncol 2020; 31: LBA85. Gadgeel SM, Pennell NA, Fidler MJ, et al. Phase II study of maintenance pembrolizumab in patients with extensive stage small cell lung cancer (SCLC). J Thorac Oncol 2018; 13: 1393-1399.

Owonikoko TK, Kim HR, Govindan R, et al. Nivolumab (nivo) plus ipilimumab (ipi), nivo, or placebo (pbo) as maintenance therapy in patients (pts) with extensive disease small cell lung cancer (ED-SCLC) after first-line (1 L) platinum-based chemotherapy (chemo): results from the double-blind, randomized phase III CheckMate 451 study. Ann Oncol 2019; 30: 1613-1621 (suppl; abstr LBA1-PR).

49 ClinicalTrials.gov. Testing the addition of radiation therapy to the usual immune therapy treatment (Atezolizumab) for extensive stage Small Cell Lung Cancer, the RAPTOR Trial. https://clinicaltrials.gov/ct2/ show/NCT04402788. Date last assessed March 4, 2021. Date last updated: June 3, 2021.

50 Reck M, Vicente D, Ciuleanu T, et al. Efficacy and safety of nivolumab (nivo) monotherapy versus chemotherapy (chemo) in recurrent small cell lung cancer (SCLC): Results from CheckMate 331. Ann Oncol 2018; 29: Suppl.10 x39-x43.

51 Ready NE, Ott PA, Hellmann MD, et al. Nivolumab monotherapy and nivolumab plus ipilimumab in recurrent small cell lung cancer: results from the CheckMate 032 randomized cohort. J Thorac Oncol 2020; 15: 426-435. Ready N, Farago AF, Braud F, et al. Third-line nivolumab monotherapy in recurrent SCLC: CheckMate 032. $J$ Thor Oncol 2019; 14: 237-244.

53 Hellmann MD, Callahan MK, Awad MM, et al. Tumor mutational burden and efficacy of nivolumab monotherapy and in combination with ipilimumab in small-cell lung cancer. Cancer Cell 2018; 33: 853-861. Ott PA, Elez E, Hiret S, et al. Pembrolizumab in patients with extensive-stage small-cell lung cancer: results from the phase Ib KEYNOTE-028 study. J Clin Oncol 2017; 35: 3823-3829.

55 Chung HC, Lopez-Martin JA, Kao SC-H, et al. Phase 2 study of pembrolizumab in advanced small-cell lung cancer (SCLC): KEYNOTE-158. J Clin Oncol 2018; 36: Suppl. 15, 8506.

56 Pujol J-L, Greillier L, Audigier-Valette C, et al. A randomized non-comparative phase II study of anti-programmed cell death-ligand 1 atezolizumab or chemotherapy as second-line therapy in patients with small-cell lung cancer: results from the IFCT-1603 trial. J Thorac Oncol 2019; 14: 903-913.

Bondarenko I, Juan-Vidal O, Pajkos G, et al. Preliminary efficacy of durvalumab plus tremelimumab in platinum-refractory/resistant ED-SCLC from Arm A of the Phase II BALTIC study. Ann Oncol 2018; 29: Suppl. 8, viii596-viii602.

$58 \mathrm{Kim}$ Y-J, Keam B, Ock C-Y, et al. A phase II study of pembrolizumab and paclitaxel in patients with relapsed or refractory small-cell lung cancer. Lung Cancer 2019; 136: 122-128.

59 Ishii H, Azuma K, Kawahara A, et al. Significance of programmed cell death-ligand 1 expression and its association with survival in patients with small cell lung cancer. $J$ Thorac Oncol 2015; 10: 426-430. Schultheis AM, Scheel AH, Ozretic L, et al. PD-L1 expression in small cell neuroendocrine carcinomas. Eur J Cancer 2015; 51: 421-426.

61 George J, Saito M, Tsuta K, et al. Genomic amplification of CD274 (PD-L1) in small-cell lung cancer. Clin Cancer Res 2017; 23: 1220-1226.

62 Park S, Lee $\mathrm{H}$, Lee B, et al. DNA damage response and repair pathway alteration and its association with tumor mutation burden and platinum-based chemotherapy in SCLC. J Thorac Oncol 2019; 14: 1640-1650.

63 Blackhall F, Frese KK, Simpson K, et al. Will liquid biopsies improve outcomes for patients with small-cell lung cancer? Lancet Oncol 2018; 19: e470-e481.

64 George J, Lim JS, Jang S, et al. Comprehensive genomic profiles of small cell lung cancer. Nature 2015; 524: 47-53.

65 Peifer M, Fernández-Cuesta L, Sos ML, et al. Integrative genome analyses identify key somatic driver mutations of small-cell lung cancer. Nat Genet 2012; 44: 1104-1110.

66 Sos ML, Dietlein F, Peifer M, et al. A framework for identification of actionable cancer genome dependencies in small cell lung cancer. Proc Natl Acad Sci U S A 2012; 109: 17034-17039.

67 Mollaoglu G, Guthrie M, Böhm S, et al. MYC drives progression of small cell lung cancer to a variant neuroendocrine subtype with vulnerability to aurora kinase inhibition. Cancer Cell 2017; 31: 270-285.

68 Sen T, Gay CM, Byers LA. Targeting DNA damage repair in small cell lung cancer and the biomarker landscape. Transl Lung Cancer Res 2018; 7: 50-68.

69 Sen T, Tong P, Stewart CA. CHK1 inhibition in small-cell lung cancer produces single-agent activity in biomarker-defined disease subsets and combination activity with cisplatin or olaparib. Cancer Res 2017; 77: 3870-3884; 
Lallo A, Frese KK, Morrow CJ, et al. The combination of PARP inhibitor Olaparib and the WEE1 inhibitor AZD1775 as a new therapeutic option for small cell lung cancer. Clin Cancer Res 2018; 24: 5153-5164.

Owonikoko TK, Dahlberg SE, Sica GL, et al. Randomized phase II trial of cisplatin and etoposide in combination with veliparib or placebo for extensive-stage small-cell lung cancer: ECOG-ACRIN 2511 study. J Clin Oncol 2019; 37: 222-229.

Lok BH, Gardner EE, Schneeberger VE, et al. PARP inhibitor activity correlates with SLFN11 expression and demonstrates synergy with temozolomide in small cell lung cancer. Clin Cancer Res 2017; 23: 523-535.

Danson SJ, Middleton MR. Temozolomide: a novel oral alkylating agent. Expert Rev Anticancer Ther 2001; 1: 13-19.

Pietanza MC, Kadota K, Huberman K, et al. Phase II trial of temozolomide with relapsed sensitive or refractory small cell lung cancer, with assessment of methylguanine-DNA methyltransferase as a potential biomarker. Clin Cancer Res 2012; 18: 1138-1145.

Palma JP, Wang YC, Rodriguez LE, et al. ABT-888 confers broad in vivo activity in combination with temozolomide in diverse tumors. Clin Cancer Res 2009; 15: 7277-7290.

Pietanza MC, Waqar SN, Krug LM, et al. Randomized, double-blind, phase II study of temozolomide in combination with either veliparib or placebo in patients with relapsed-sensitive or refractory small-cell lung cancer. J Clin Oncol 2018; 36: 2386-2394.

Farago AF, Yeap BY, Stanzione M, et al. Combination olaparib and temozolomide in relapsed small-cell lung cancer. Cancer Discov 2019; 9: 1372-1387.

Tumini E, Herrera-Moyano E, San Martín-Alonso M, et al. The antitumor drugs trabectedin and lurbinectedin induce transcription-dependent replication stress and genome instability. Mol Cancer Res 2019; 17: 773-782.

Trigo J, Subbiah V, Besse B, et al. Lurbinectedin as second-line treatment for patients with small-cell lung cancer: a single-arm, open-label, phase 2 basket trial. Lancet Oncol 2020; 21: 645-654.

Farago AF, Drapkin BJ, Lopez-Vilarino de Ramos JA, et al. ATLANTIS: A phase III study of lurbinectedin/ doxorubicin versus topotecan or cyclophosphamide/doxorubicin/vincristine in patients with small-cell lung cancer who have failed one prior platinum-containing line. Future Oncol 2019; 15: 231-239.

Antony J, Huang RYJ. AXL-driven EMT state as a targetable conduit in cancer. Cancer Res 2017; 77 : 3725-3732.

Balaji K, Vijayaraghavan S, Diao L, et al. AXL inhibition suppresses the DNA damage response and sensitizes cells to PARP inhibition in multiple cancers. Mol Cancer Res 2017; 15: 45-58.

, Tong P, Diao L, et al. Targeting AXL and MTOR pathway overcomes primary and acquired resistance to WEE1 inhibition in small-cell lung cancer. Clin Cancer Res 2017; 23: 6239-6253.

Murai J, Feng Y, Yu GK, et al. Resistance to PARP inhibitors by SLFN11 inactivation can be overcome by ATR inhibition. Oncotarget 2016; 7: 76534-76550.

Parsels LA, Karnak D, Parsels JD, et al. PARP1 trapping and DNA replication stress enhance radiosensitization with combined WEE1 and PARP inhibitors. Mol Cancer Res 2018; 16: 222-232.

Laird JH, Lok BH, Ma J, et al. Talazoparib is a potent radiosensitizer in small cell lung cancer cell lines and xenografts. Clin Cancer Res 2018; 24: 5143-5152.

Cui M, Augert A, Rongione M, et al. PTEN is a potent suppressor of small cell lung cancer. Mol Cancer Res 2014; 12: 654-659.

Dabir S, Babakoohi S, Kluge A, et al. RET mutations and expression in small-cell lung cancer. $J$ Thorac Oncol 2014; 9: 1316-1323.

Schultheis AM, Bos M, Schmitz K, et al. Fibroblast growth factor receptor 1 (FGFR1) amplification is a potential therapeutic target in small-cell lung cancer. Mod Pathol 2013; 27: 214-221.

Poirier JT, Gardner EE, Connis N, et al. DNA methylation in small cell lung cancer defines distinct disease subtypes and correlates with high expression of EZH2. Oncogene 2015; 34: 5869-5878.

Gardner EE, Lok BH, Schneeberger VE, et al. Chemosensitive relapse in small cell lung cancer proceeds through an EZH2-SLFN11 axis. Cancer Cell 2017; 31: 286-299.

Koinis F, Agelaki S, Karavassilis V, et al. Second-line pazopanib in patients with relapsed and refractory small-cell lung cancer: a multicentre phase II study of the Hellenic Oncology Research Group. Br J Cancer 2017; 117: 8-14.

Russo A, Ron DA, Rasschaert M, et al. Is there room for personalized medicine in small-cell lung cancer (SCLC)? Remarkable activity of pazopanib in refractory FGFR1-amplified ED-SCLC? JCO Precision Oncol 2019; 3: 1-8.

Keen N, Taylor S. Aurora-kinase inhibitors as anticancer agents. Nat Rev Cancer 2004; 4: 927-936.

Melichar B, Adenis A, Lockhart AC, et al. Safety and activity of alisertib, an investigational aurora kinase A inhibitor, in patients with breast cancer, small-cell lung cancer, non-small-cell lung cancer, head and neck squamous-cell carcinoma, and gastro-oesophageal adenocarcinoma: a five-arm phase 2 study. Lancet Oncol 2015; 16: 395-405.

Owonikoko TK, Niu H, Nackaerts K, et al. Randomized phase II study of paclitaxel plus alisertib versus placlitaxel plus placebo as second-line therapy for SCLC: primary and correlative biomarker analyses. J Thorac Oncol 2020; 15: 274-287. 
Goodman AM, Sokol ES, Frampton GM, et al. Microsatellite-stable tumors with high mutational burden benefit from immunotherapy. Cancer Immunol Res 2019; 7: 1570-1573.

98 Jiao S, Xia W, Yamaguchi $\mathrm{H}$, et al. PARP inhibitor upregulates PD-L1 expression and enhances cancer-associated immunosuppression. Clin Cancer Res 2017; 23: 3711-3720.

99 Sen T, Rodriguez BL, Chen L, et al. Targeting DNA damage response promotes antitumor immunity through STING-mediated T-cell activation in small cell lung cancer. Cancer Discov 2019; 9: 646-661.

100 Tanaka K, Isse K, Fujihira T, et al. Prevalence of Delta-like protein 3 expression in patients with small cell lung cancer. Lung Cancer 2018; 115: 116-120.

101 Rudin CM, Pietanza MC, Bauer TM, et al. Rovalpituzumab tesirine, a DLL3-targeted antibody-drug conjugate in recurrent small cell lung cancer; a first-in-human, first-in-class, open-label, phase 1 study. Lancet Oncol 2017; 18: 42-51.

102 Morgensztern D, Besse B, Greillier L, et al. Efficacy and safety of Rovalpituzumab Tesirine in third-line and beyond patients with DLL3-expressing, relapsed/refractory small-cell lung cancer: results from the phase II TRINITY Study. Clin Cancer Res 2019; 25: 6958-6966.

103 Blackhall F, Jao K, Greillier L, et al. Efficacy and safety of Rovalpituzumab Tesirine compared with topotecan as second-line therapy in DLL3-high small cell lung cancer: results from the phase 3 TAHOE study. $J$ Thorac Oncol 2021; in press [https://doi.org/10.1016/j.jtho.2021.02.009].

104 ClinicalTrials.gov. A randomized, double-blind, placebo-controlled phase 3 study of rovalpituzumab tesirine as maintenance therapy following first-line platinum-based chemotherapy in subjects with extensive stage small cell lung cancer (MERU). https://clinicaltrials.gov/ct2/show/NCT03033511. Date last accessed March 4, 2021. Date last updated: December 21, 2020.

105 Borromeo MD, Savage TK, Kollipara RK, et al. ASCL1 and NEUROD1 reveal heterogeneity in pulmonary neuroendocrine tumors and regulate distinct genetic programs. Cell Rep 2016; 16: 1259-1272.

106 Rudin CM, Poirier JT, Byers LA, et al. Molecular subtypes of small cell lung cancer: a synthesis of human and mouse model data. Nat Rev Cancer 2019; 19: 289-287.

107 Baine MK, Hsieh MS, Lai V, et al. SCLC subtypes defined by ASCL1, NEUROD1, POU2F3, and YAP1: a comprehensive immunohistochemical and histopathologic characterization. J Thorac Oncol 2020; 15: 1823-1835.

108 Ireland AS, Micinski AM, Kastner DW, et al. MYC drives temporal evolution of small cell lung cancer subtypes by reprogramming neuroendocrine fate. Cancer Cell 2020; 38: 60-78.e12.

109 Malhotra J, Nikolinakos P, Leal T, et al. A phase I/II study of Rovalpituzumab Tesirine in combination with nivolumabıipilimumab in patients with previously treated extensive-stage small cell lung cancer. $J$ Thorac Oncol 2021; in press [https://doi.org/10.1016/j.jtho.2021.02.022].

110 Gay CM, Stewart A, Park EM, et al. Patterns of transcription factor programs and immune pathway activation define four major subtypes of SCLC with distinct therapeutic vulnerabilities. Cancer Cell 2021; 39: 346-360. 